\title{
Anspruchsvolles Leitprojekt
}

\section{Die ökologische Modernisierung von Wirtschaft und Gesellschaft voranbringen, das hatte die rot-grüne Bundesregierung als eines der Hauptziele im Koalitions- vertrag vereinbart. Die Arbeitsgruppe Ökologischer Strukturwandel der VöW hat im Rahmen einer Artikelserie in der Frankfurter Rundschau einige Aspekte und Akteure einer erfolgreichen ökologischen Modernisierung näher untersucht und eine Zwischenbilanz gezogen. Der Beitrag fasst wesentliche Punkte zusam- men (1).}

$\mathrm{I}$ Von Annette Volkens und Jan Nill für die AG Ökologischer Strukturwandel Anbetracht der bestehenden Umweltprobleme und deren drohender Folgen ist eine ökologische Modernisierung dringend nötig. Es sind zwar bei einigen gut wahrnehmbaren Umweltproblemen wie der Luftverschmutzung in den letzten Jahren erhebliche Fortschritte erzielt worden. Viele Probleme wie Klimaveränderungen, Flächenverbrauch oder Artensterben sind jedoch weniger sichtbar und gehen eher schleichend vonstatten. Hier sind die derzeitigen Tendenzen deutlich negativ. Eine Chance zur Trendumkehr bietet die ökologische Modernisierung von Wirtschaft und Gesellschaft.

Ökologische Modernisierung heißt zunächst, den Faktor Umwelt bewusst in das Handlungskalkül aller Akteure aufzunehmen, die Umweltkosten sichtbar zu machen und dabei auch alle wirtschaftlichen Potenziale sowie Arbeitsplatz- und Innovationschancen des Umweltschutzes zu nutzen. (2). Es geht darum, dem Markt verlässliche ökologische Rahmenbedingungen zu setzen. Ökonomische und kommunikative Instrumente statt nur Ge- und Verbote sowie integrierte Technologien statt End-of-Pipe-Lösungen lauten die bekannten, aber immer noch aktuellen Schlagworte. Doch ökologische Modernisierung bedeutet mehr. Es geht gleichermaßen darum, dem Querschnittscharakter von Umweltproblemen auch im politischen Alltag gerecht zu werden und alle gesellschaftlichen Akteure, insbesondere Unternehmen, Nichtregierungsorganisationen und Verbraucher, einzubeziehen. Stichworte sind hier Politikintegration, Öko-Effizienz und Veränderungen des Konsumverhaltens. Umweltschutz bedeutet dabei nicht zwingend Verzicht auf individuelle Freiheiten. Die Verbesserung der Umweltsituation bringt auch eine Steigerung der Lebensqualität für heutige und zukünftige Generationen mit sich.

\section{Die Rolle der Politik ...}

Dabei spielt die Politik als rahmensetzender Akteur für eine ökologische Modernisierung weiterhin eine bedeutende Rolle, denn der Markt allein kann die bestehenden Umweltprobleme nicht lösen. Ein zentrales Instrument bleibt die Ökologische Steuerreform, weil sie langfristig alle Sektoren und Handlungsfelder betrifft und beeinflusst. Auch bei den wichtiger werdenden globalen Umweltproblemen wie Klimaschutz ist ein nationaler Beitrag unerlässlich. Doch neben geeigneten Instrumenten geht es vor allem um eine Veränderung der politischen Herangehensweise: Der Faktor Umwelt muss in die Entscheidungsfindung aller politischen Bereiche integriert werden. Beispielsweise müssen Umwelt- und Verkehrspolitik in die gleiche Richtung wirken und sich nicht, wie heute Alltag, widersprechen. Außerdem sind neue Ansätze der Innovationsförderung gefragt, die einen Ausweg aus dem Dilemma bieten, dass politische Maßnahmen oft entweder ökologisch nicht wirksam oder aber gesellschaftlich und politisch nicht durchsetzbar erscheinen

\section{D... und weiterer Akteure}

Aber auch die Eigeninitiative anderer Akteure ist gefordert. Nachdem die Wirtschaft bei der Verringerung des Ressourcenverbrauches und der Energieeinsparung in der Produktion erhebliche Erfolge erzielt hat, ist sie in Zukunft bei Produktinnovationen sowie als aktive Gestalterin der Modernisierung gefordert. Dies betrifft vor allem ihren Einfluss auf die Konsumstile. Sowohl durch die angebotenen Produkte und deren Verpakkung als auch durch eine weit reichende Information zu Inhaltsstoffen, Wirkungen etc. üben Unternehmen Einfluss auf Art und Menge des Konsums aus.
Eine bedeutsame Rolle spielen Umweltverbände und Bürgerinitiativen. Konfliktstrategien der Nichtregierungsorganisationen (NGOs) sind oft medienwirksam, heben bestehende Umweltprobleme deutlich hervor und rücken sie damit in das Bewußtsein aller Bevölkerungsschichten. Immer wichtiger für NGOs werden Kooperationen mit Unternehmen oder die Beratung politischer Entscheidungsträger, um ausgehend von bestehenden Strukturen Umweltaspekte stärker einzubringen und die öffentliche Unterstützung hierfür zu verstärken.

Von den Bürgern als Verbraucher allein ist allerdings nur ein Teilbeitrag zur ökologischen Modernisierung zu erwarten. Dies liegt neben veränderten Lebensstilen auch am Charakter heutiger Umweltprobleme. Diese sind im Gegensatz zur Luft- und Gewässerverschmutzung, oft nicht direkt sichtbar. Daher werden auch die Erfolge individueller Anstrengungen zum Schutz der Umwelt nicht mehr direkt spürbar und die Motivation für den bzw. des Einzelnen immer schwieriger. Vorreiter bei ökologisch verträglicheren Konsumstilen bekommen dies in ihrem Umfeld zu spüren. Verhaltensänderungen können daher nur breiten Erfolg haben, wenn Lebensstile und -umfeld richtig einbezogen werden. Aber auch als bewusst nach ethisch-ökologischen Kriterien anlegende Kapitalanleger gewinnen die Haushalte an Bedeutung, wenngleich auch hier veränderte Rahmenbedingungen wie Informationspflichten, steuerliche Vorteile und eindeutige Bewertungsmaßstäbe förderlich wären.

Schließlich spielen Bildung und Wissenschaft eine wichtige Rolle, um gleichzeitig das Bewusstsein der Bevölkerung für die Wichtigkeit des Themas zu stärken und innovative Ansätze für die Umsetzung der ökologischen Modernisierung zu forcieren. Hier ist neben entsprechenden Forschungsansätzen insbesondere die Multiplikatorenfunktion der Hochschulen bei der Ausbildung von Führungskräften für Wirtschaft, Gesellschaft und Wissenschaft gefragt.

\section{Rot-grüne Zwischenbilanz}

So entscheidend also der Beitrag aller genannten Akteure ist, so wird doch immer wieder die besondere Rolle der Politik beim Vorantreiben und der Koordination des Prozesses einer ökologischen Modernisierung deutlich. Wie sieht vor diesem Hintergrund die Zwischenbilanz der rotgrünen Bundesregierung aus?

Bisher ist die ökologische Modernisierung nicht als Leitmotiv der Politik von Rot-Grün erkennbar: 
Die Bundesregierung hat zwar eine Vielzahl einzelner Schritte auf dem Weg zur ökologischen Modernisierung unternommen, Beispiele sind die Ökologische Steuerreform sowie Maßnahmen zur Verbesserung der Energieeffizienz und einer umweltverträglicheren Energieerzeugung. Vieles scheint jedoch losgelöst voneinander erdacht, die Ministerien scheinen teilweise gegeneinander statt miteinander zu arbeiten. Dies wird unter anderem an Subventionen und Steuererleichterungen für die Wirtschaft, den Kohlebergbau und die Landwirtschaft deutlich, die den Zielen der Ökosteuer zuwider laufen. Die Entfernungspauschale stellt zwar den ÖPNV dem Auto gleich und sorgt so für ein Ende der Bevorzugung des Autoverkehrs, steht durch die gleichzeitige Erhöhung der Pauschale pro Kilometer für Fernpendler aber im Widerspruch zur ökologisch wichtigen Verringerung des Flächenverbrauchs - sie subventioniert letztlich auch weiterhin das Wohnen im Grünen und den damit verbundenen Pendlerstrom mit all seinen Folgen.

Dass ein erfolgreiches Zusammenspiel der Ressorts möglich ist, zeigt das im Oktober verabschiedete Klimaschutzprogramm. Es legt für unterschiedliche Bereiche, wie etwa Verkehr oder Bauen und Wohnen, sektorale Ziele zur Kohlendioxid-Reduktion fest. Dies ist auch dringend nötig, wenn das Reduktionsziel von 25 Prozent bis 2005 noch erreicht werden soll: während sich die Emissionen im Industriesektor - auch durch das Absterben energieintensiver Wirtschaftszweige in den neuen Bundesländern nach der Wiedervereinigung - deutlich verringert haben, zeigt der Trend bei den Haushalten und vor allem beim Verkehr deutlich in die entgegengesetzte Richtung.

Akzente hat Rot-Grün bisher vor allem bei der Energieversorgung gesetzt, eine echte Energiewende wird mit den bislang beschlossenen Einzelmaßnahmen jedoch noch nicht erreicht werden können. Die Energiewende ist aber notwendig, um auch über 2005 hinaus weiterhin zur Entlastung der Umwelt beizutragen. Ein bedeutender Schritt in diese Richtung wäre eine bereits in dieser Legislaturperiode beschlossene konsequente Weiterführung der Ökologischen Steuerreform über das Jahr 2003 hinaus. Die Korrektur bisheriger Mängel, wie die erheblichen Ausnahmen für die Industrie sowie die Nichtbefreiung erneuerbarer Energien, sollte dabei mit dem generellen Abbau kontraproduktiver Subventionen verknüpft werden.
Erhebliche Anstrengungen sind auch im Verkehrsbereich notwendig; dies gilt für den Personen- wie Güterverkehr. Energieeffizientere Technik und verringerter Schadstoffausstoß werden durch ein stetig steigendes Aufkommen konterkariert. Ansatzpunkte sind die Förderung umweltfreundlicher öffentlicher Verkehrsträger ebenso ein wie ein Umdenken in der Siedlungspolitik. Um sowohl Personen- als auch Güterverkehr von der Straße auf die Schiene umzulenken, ist eine übergreifende Infrastrukturpolitik zugunsten der Schiene nötig, flankiert durch die konsequente Einführung der leistungsabhängigen Schwerverkehrsabgabe im Güterverkehr.

Die letzten Jahre haben aber auch gezeigt, wie schwierig es für die Regierung ist, Projekte für die ökologische Modernisierung der Bevölkerung zu vermitteln. Exemplarisch zeigt dies die immer wieder aufflammende Debatte um die Benzinpreise. Hier wird deutlich: Für weitere Fortschritte bei der ökologischen Modernisierung geht es sowohl um die Setzung entsprechender Rahmenbedingungen als auch um geeignete Instrumente und Institutionen zur Vermittlung und Aktivierung, damit sich die anderen Akteure aus eigenem Antrieb aktiv am Projekt der ökologischen Modernisierung beteiligen.

\section{- Zukunftsperspektiven}

Weiterfuihrend könnte hier die Entwicklung einer konsistenten, mit Zielen und Maßnahmen unterlegten nationalen Nachhaltigkeitsstrategie sein. Sie könnte ein Leitbild für eine auf ökologische Modernisierung ausgerichtete Politik formulieren sowie als Orientierung für die Aktivitäten aller anderen Betroffenen dienen. Bisher wurde jedoch mit der Einsetzung eines Staatssekretärsausschusses und dem geplanten Rat für nachhaltige Entwicklung nur der institutionelle Rahmen für eine solche Strategie festgelegt. Während die inhaltliche Ausgestaltung noch aussteht, sind auch die Einflussmöglichkeiten der neu geschaffenen Institutionen bisher nicht deutlich erkennbar. Hier geht es - wie skizziert um ein Bündel von rahmensetzenden Maßnahmen unter anderem in den Bereichen Energie und Verkehr, aber auch um Leitbilder und Ansatzpunkte, die zum Beispiel die VerbraucherInnen direkt ansprechen und ohne Zeigefinger und die Forderung nach Verzicht zu einem Umdenken in der Gesellschaft führen. Beispielhaft genannt werden kann hier das Projekt „,Viel Umwelt für wenig Geld“ der Arbeiterwohlfahrt. Haushalte mit geringem Einkommen erproben Maßnahmen, die zu einer Entlastung der Umwelt führen, die Lebensqualität stei-

\section{AG Ökologischer Strukłurwandel}

Die Arbeitsgruppe „Ökologischer Strukturwandel" der Vereinigung für ökologische Wirtschaftsforschung (VÖW) e.V. ist ein auch für Nicht-VÖW-Mitglieder offenes Netzwerk von Menschen, die mit unterschiedlichen Aktivitäten die Ökologisierung von Wirtschaft und Gesellschaft unterstützen möchten. Die Beteiligten sind in Unternehmen und gesellschaftlichen Organisationen, Ministerien und Parlamenten, Universitäten und freien Forschungsinstituten tätig. Hauptdiskussionsmedium ist ein AG-eigener Listserver (voew-sw@listserv.uni-oldenburg.de). Weitere Mitstreiterlnnen sind jederzeit willkommen.

Kontakt: oesw@voew.de

gern und gleichzeitig Kosten sparen. Die Teilnehmer geben ihre Erfahrungen an Freunde und Bekannte weiter und sorgen so für die Ausweitung eines umweltgerechteren Konsumstils.

Insgesamt zeigt sich, dass eine ökologische Modernisierung im Sinne von zukunftsfähigen Innovationen und Verhaltensänderungen nur gelingen kann, wenn sie von Politik, Wirtschaft und Gesellschaft getragen wird, die Interessenkonflikte zwischen den Betroffenen sowie den verschiedenen Verbänden produktiv aufgenommen werden und Synergien aus den bei den verschiedenen Akteuren vorhandenen Potenzialen genutzt werden. Zentral bleibt dabei die Langfristigkeit der Perspektive, die versucht, den Prozess so zu institutionalisieren, dass er über Legislaturperioden und Einzelereignisse hinaus trägt.

\section{Anmerkungen}

(1) Die gesammelten Beiträge der Serie werden von der VöW in einer eigenen Publikation veröffentlicht, die bei der VöW-Geschäftsstelle bezogen werden kann.

(2) Im Gegensatz zur engen Fassung der ökologischen Modernisierung bei Martin Jänicke (vgl. Ökologisches Wirtschaften 6/2000, S. 27-28) wird Ökologische Modernisierung von der AG breiter verstanden und schlieft insbesondere auch Verhaltensänderungen mit ein. Sie kann daher je nach Reichweite durchaus zu einem ökologischen Strukturwandel führen.

\section{Die Autorlnnen}

Annette Volkens ist Pressesprecherin der Vereinigung für ökologische Wirtschaftsforschung (VÖW), Jan Nill ist wissenschaftlicher Mitarbeiter des Instituts für ökologische Wirtschaftsforschung und Mitglied des vöW-Vorstands.

Kontakt: VÖW e.V., Geschäftsstelle, Potsdamer Str. 105, 10785 Berlin. Tel. 030/8851800, Fax 030/ 8825439,E-mail oesw@voew.de, Annette.Volkens@ voew.de, Jan.Nill@ioew.de 
(c) 20I0 Authors; licensee IÖW and oekom verlag. This is an article distributed under the terms of the Creative Commons Attribution Non-Commercial No Derivates License (http://creativecommons.org/licenses/by-nc-nd/3.o/), which permits unrestricted use, distribution, and reproduction in any medium, provided the original work is properly cited. 